\title{
Les transformations dynamiques des configurations de participation formatives en situation de travail : le cas des ergothérapeutes Dynamic transformations of vocational training participation frameworks at work: The case of occupational therapists
}

\author{
Renaud Janet ${ }^{1}$ et Vasiliki Markaki-Lothe ${ }^{2}$ \\ ${ }^{1}$ Ergothérapeute, formateur, chercheur-indépendant, France \\ ${ }^{2}$ Laboratoire de Recherche sur les Apprentissages en Contexte (LaRAC), Université Grenoble Alpes, \\ France
}

\begin{abstract}
Résumé. Un tiers du temps de formation des ergothérapeutes se déroule en stage. Cependant il n'existe aucune recommandation didactique pour l'encadrement et le suivi des stagiaires. Cette question émerge : Quelles sont les modalités pratiques de l'accompagnement tutoral dans l'acquisition des compétences en stage ? Nous proposons, ici, d'explorer des enregistrements audio-vidéo de pratiques tutorales en situation de travail réel couplés à des entretiens semi-directifs auprès des tuteurs. Les données sont analysées selon les principes analytiques de l'Analyse Conversationnelle issue de l'ethnométhodologie. En mettant en relation les modalités de participation des acteurs avec les enjeux et implications de la formation professionnelle des ergothérapeutes, nous repérons le caractère dynamique et poreux de quatre configurations de participation typiques de la formation: observation, coanimation, monstration et laisser-faire.
\end{abstract}

\begin{abstract}
One third of the training time for occupational therapists is spent on practice. However, there are no didactic recommendations for the supervision and monitoring of trainees. This question rises: What are the practical modalities of tutorial support in the acquisition of competences during the internship? We propose, here, to explore audio-video recordings of tutorial practices in real work situations coupled with semi-directive interviews with tutors. The data are analysed according to the analytical principles of Conversational Analysis from ethnomethodology. By relating the participation modalities of the actors with the stakes and implications of the professional training of occupational therapists, we identify the dynamic and porous character of four configurations of participation characterized as typical of the vocational training: observation, co-animation, demonstration and laisser-faire.
\end{abstract}




\section{Introduction}

À ce jour, un tiers du temps de la formation des ergothérapeutes se déroule en stage et les deux tiers dans un institut de formation d'ergothérapeute (IFE). L'encadrement formatif de stage est fait par les tuteurs ergothérapeutes professionnels. Ceux-ci sont chargés de l'accompagnement de l'étudiant, l'évaluation du stage et des compétences. Avant le début d'un stage ou lors de la première semaine, l'étudiant doit négocier ses objectifs de stage avec son tuteur en relation avec les activités professionnelles du stage et les compétences à acquérir pour sa formation. Le parcours de formation en stage est référencé dans un document unique appelé PORTFOLIO qui sert de guide au tuteur et de référence pour le suivi pédagogique de l'étudiant.

Le Syndicat des Instituts de Formation en Ergothérapie Français (SIFEF) qui regroupe l'ensemble des instituts de formation en ergothérapie en France souligne que : "Le formateur en IFE et le tuteur professionnel aident à l'acquisition d'une démarche de soins et d'interventions en rapport avec les interactions personne - activité - environnement et permet l'exercice d'un raisonnement inductif, analogique ou déductif ${ }^{1}$. Cette déclaration met en évidence le caractère hybride des modalités pédagogiques de la formation des ergothérapeutes, à la fois en Institut de formation et en situation de travail, visant le développement de compétences complexes du métier en lien avec les soins et les interventions spécifiques auprès de patients ayant des diagnostics et donc des besoins fort différents.

Si l'organisation de la formation permet à l'étudiant de réaliser plusieurs stages et le confronte à la réalité et donc à la difficulté du terrain, les lieux d'accueil des étudiants se confrontent aussi à la difficulté de former efficacement de nouveaux collègues. En 2010, Jovic, Goldszmidt et Monguillon concluent que la gestion administrative des stagiaires dans un établissement de soin correspond en moyenne à la mobilisation d'un professionnel pendant trois heures et demie par jour durant toute l'année. Ils arrivent à évaluer aussi que le temps moyen d'encadrement direct est de 50 minutes à 143 minutes par jour par étudiant. Or, les lieux d'accueils ont rarement la possibilité de détacher des professionnels pour la seule fonction des tuteurs. L'accompagnement des stagiaires doit se faire donc en même temps que la prise en charge de patients et l'activité réelle du stage est soumise à une double contrainte : former et en même temps travailler efficacement auprès des patients.

Dans la littérature, l'activité tutorale en ergothérapie n'a pas été beaucoup documentée. Elle a surtout été abordée dans des revues professionnelles spécialisées et explorée essentiellement à l'aide d'entretiens auprès des professionnels, étudiant par exemple les perceptions des professionnels, les dimensions identitaires et les ressources mobilisées dans le cadre de l'accompagnement des étudiants en ergothérapie (Gosselin, Dutil, 1995).

Or, de plus en plus dans le champ des sciences du travail et de la formation, des travaux soulignent l'intérêt des études descriptives des interactions humaines en situation pour mieux comprendre les mécanismes sous-jacents à l'activité, des études qui montrent non pas ce que les gens pensent mais ce qu'ils font (Filliettaz et al., 2021). Aborder l'activité de la formation depuis la perspective de ce qui est observable et peut être décrit, offre un nouvel éclairage à la manière dont s'organisent les actions entreprises pour montrer, transmettre, évaluer. Cet article, s'intéresse justement à une description systématique des interactions réelles qui accomplissent l'activité tutorale en lieu de stage d'ergothérapie. Avec une approche constructiviste et inductive, fortement inspirée de l'analyse conversationnelle issue de l'ethnométhodologie (Sacks, 1992), plusieurs questions émergent : Quelles sont les modalités pratiques de l'accompagnement tutoral dans l'acquisition des compétences en stage ? À

\footnotetext{
${ }^{1}$ https://www.sifef.fr/formation/
} 
l'aide de quelles ressources pratiques cet accompagnement est-il réalisé et comment est-il repérable par les participants ? Comment les participants aux interactions tutorales négocientils leurs rôles respectifs et leur légitimité de participation lors des événements interactionnels ?

Dans ce qui suit nous allons apporter des précisions concernant le contexte de notre étude et le cadre théorique et méthodologique mobilisé. Nous allons ensuite présenter les résultats de nos analyses avant de conclure.

\section{Contexte}

\subsection{La population}

La population dans cette étude est composée des professionnels et stagiaires de deux services d'ergothérapie d'un même établissement. Un service est orienté sur la prise en soin de jeunes patients ayant des troubles psychiatriques et le second est un service d'ergothérapie de soin de suite et de réadaptation spécialisé en neurologie centrale (SSR). Il y avait deux périodes de captation de données, l'une pour le service de SSR et la seconde pour le service de psychiatrie. La captation des données vidéo s'est déroulée sur huit mois. Au total, il y avait eu trois ergothérapeutes en interaction avec des patients et des stagiaires. Il y avait deux étudiantes, l'une était en fin de deuxième année et l'autre en fin de troisième année. Concernant les patients filmés, 26 patients ont été filmés mais n'ont pas fait pas l'objet d'une étude particulière. Après avis du Comité de Protection des Personnes, qui a considéré que notre recherche ne faisait pas parti de la règlementation de la loi Jardé, les personnes filmées ont signé les autorisations légales de droit à l'image et ont donné leur consentement pour l'exploitation de ces données dans le cadre de cette recherche et de sa diffusion.

Concernant les critères d'inclusion nous avons ciblé des services d'ergothérapie accueillant des stagiaires sur la période de Novembre 2017 à Juin 2018 sur le bassin Grenoblois. Les ergothérapeutes filmés en interaction avec les étudiants en formation devaient avoir au moins une année de pratique professionnelle. Concernant les critères d'exclusion, ils étaient liés à l'acceptation ou non d'une personne d'être filmée. Sur le document de consentement éclairé, la personne pouvait aussi inscrire des critères spécifiques de non exploitation des données vidéo. En raison de la durée relativement courte de l'étude, nous avons réalisé en plus des captures vidéo, des entretiens avec quatre ergothérapeutes, pour recueillir leur point de vue sur la formation des stagiaires en situation de travail. Parmi les ergothérapeutes avec lesquels nous nous étions entretenus, deux ont été filmés lors des captations vidéo et deux n'ont pas été filmés mais avaient une expérience professionnelle dans l'encadrement de stagiaires.

\subsection{Le cadre conceptuel et les méthodes d'analyse}

Dans le cadre de cette recherche, nous avons donc exploré des enregistrements audiovisuels de pratiques tutorales en situation de travail réel couplés à des entretiens semi-directifs auprès des tuteurs.

En nous appuyant sur le cadre méthodologique et conceptuel de l'Analyse Conversationnelle issue de l'ethnométhodologie (Sacks, Schegloff \& Jefferson, 1974; Schegloff \& Sacks, 1973; Schegloff, Jefferson \& Sacks, 1977; Sacks, 1992; Schegloff, 2007) qui a inspiré plusieurs travaux sur les interactions professionnelles et didactiques (Filliettaz, Durand et al., à paraître; Markaki-Lothe, Lelarge, 2020 ; Pekarek, 2000) nous avons focalisé notre attention sur l'accomplissement pratique et dynamique de l'accompagnement tel qu'il émerge en contexte, en tenant compte des détails de l'interaction vers lesquels s'orientent les professionnels, les formateurs et les apprenants dans l'action. 
Dans un premier temps, l'analyse des données audiovisuelles a consisté à une retranscription fine de tous les échanges verbaux et du comportement non verbal des participants visible sur les vidéos. Les transcriptions nous ont permis dans un deuxième temps, de reconstruire pas à pas les modalités observables de participation des tuteurs et des stagiaires en présence d'un patient dans l'activité prévue, moment par moment, action par action.

Cette reconstruction réalisée en se plaçant du point de vue des participants, a mis en lumière des récurrences organisationnelles dans l'accomplissement interactionnel de l'accompagnement. Toutes les récurrences, impliquant des interactions tripartites entre tuteur, stagiaire et patient, ont été extraites et regroupées dans une collection. Les éléments de cette collection ont été comparés entre eux pour pouvoir identifier des constances et des variations langagières et comportementales selon trois critères (voir Filliettaz, Rémery et Trébert, 2014) :

- un critère praxéologique, qui tient compte des orientations des participants vers l'action et la manière dont ils rendent visible aux autres participants leur compréhension.

- un critère identitaire, à travers la définition locale des rôles qu'endossent les participants dans l'action. Ces rôles ne correspondent pas toujours aux statuts des participants.

- un critère communicationnel, qui considère les ressources langagières verbales et non verbales que les participants mobilisent pour se coordonner et s'engager dans l'action.

Cette comparaison a donné lieu à un nouveau classement permettant de caractériser l'accompagnement tutoral effectif observé.

Les entretiens semi-directifs avec les professionnels, conduits dans un second temps après les premières analyses, nous ont permis d'affiner nos interprétations et mettre en relation nos résultats avec les enjeux et implications de la formation professionnelle des ergothérapeutes.

\section{Analyse et résultats}

Nos analyses ont mis en évidence l'existence de quatre configurations interactionnelles de participation récurrentes dans nos données et leurs spécificités : quand le tuteur observe le travail du stagiaire ou l'observation, quand le tuteur et le stagiaire travaillent ensemble avec le patient ou la co-animation, quand le tuteur montre au stagiaire comment réaliser une tâche ou la monstration et enfin quand le tuteur laisse le stagiaire agir seul et s'en éloigne ou le «laisser-faire ». Ces configurations, bien qu'elles soient annoncées comme typiques de la formation professionnelle accompagnée (Kunégel, 2011 ; Filliettaz, Rémery et Trébert, 2014), ont été peu documentées dans la littérature en situation de travail réel. À notre connaissance, la formation des ergothérapeutes n'a d'ailleurs jamais fait l'objet d'une telle recherche auparavant. Notre travail vise alors à documenter les modalités d'accomplissement sous-jacentes à ces quatre formes d'accompagnement typiques dans la formation professionnelle, chargés d'enjeux didactiques, dans un nouveau contexte de travail. Il vise aussi de les penser à travers les problèmes pratiques auxquels font face les professionnels des métiers de service quand ils réalisent ces configurations. Les quatre configurations interactionnelles de participation identifiées sont ainsi abordées non pas comme des catégories d'activités isolées mais à partir du contexte interactionnel de l'activité en train de se faire dans lequel elles sont initiées et se transforment. 


\subsection{L'initiation d'une séquence d'observation}

L'activité d'observation est une activité de formation où le tuteur observe et évalue le stagiaire qui est dans une situation de mise au travail assistée. Dans l'extrait qui suit, la stagiaire va pouvoir investir un espace mis en place par la référente pour lui permettre de réaliser l'activité professionnelle en autonomie sous le contrôle de cette dernière. Cependant en présence de la patiente, et en étant donc en pleine activité professionnelle, passer le relais ne se fait jamais de manière explicite et directe. Voici un exemple qui illustre cette situation. L'ergothérapeute référente (REF) et la stagiaire (STA) interagissent avec la patiente (LEA). Arthur (ART) est un autre patient au fond de la salle.

\section{Extrait 1 : «Couture_a »}

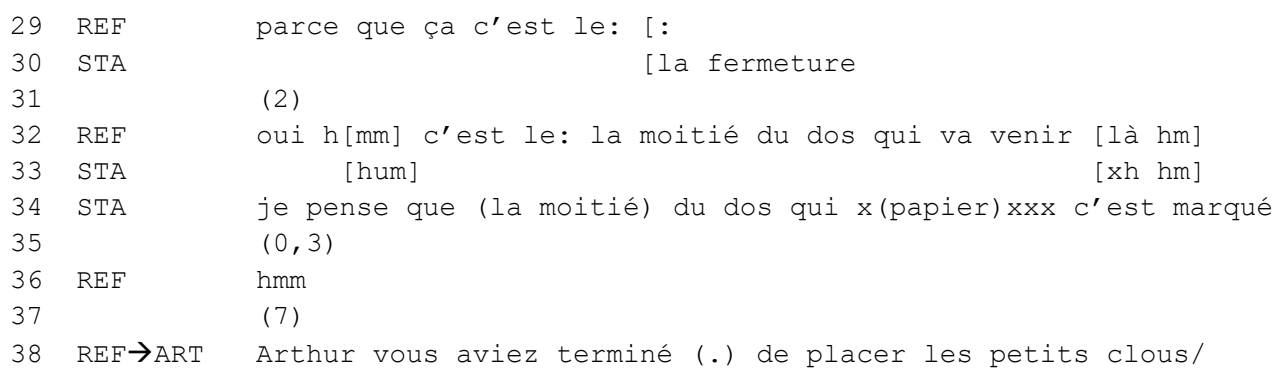

Dans cet extrait REF formule le début d'une question à haute voix concernant les pièces de tissus (ligne 29). En même temps elle manipule ces pièces en observant ce qui est écrit dessus (lignes non montrées ici) (Image 1).

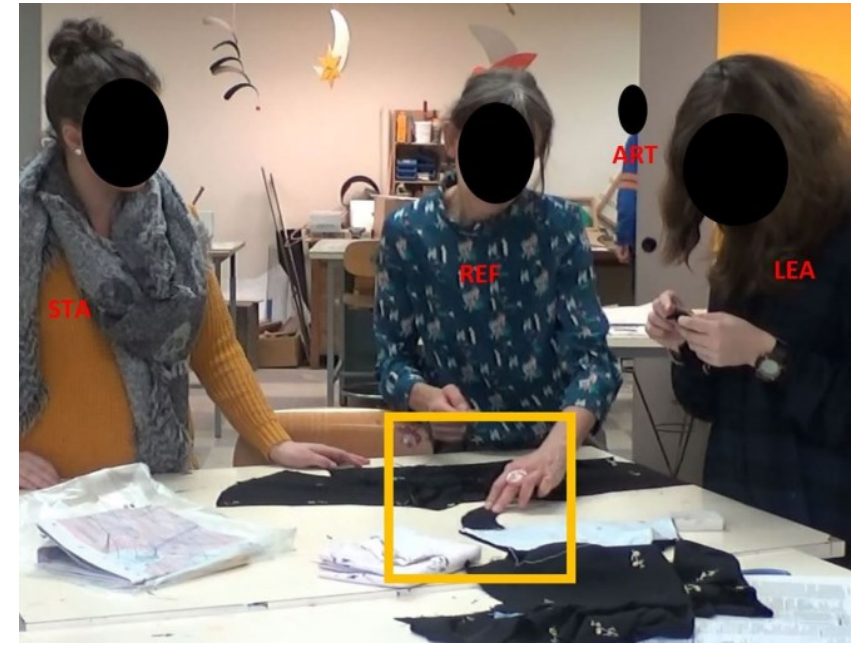

Image 1. Séquence d'observation A.

La question n'est pas explicitement adressée à STA ou LEA et rétrospectivement nous nous rendons compte que de cette manière elle offre une opportunité à la stagiaire, sans l'imposer, de prendre la place de celle qui sait faire et d'accompagner la patiente. STA se saisit en effet de cette opportunité et répond à la question (ligne 30). En manipulant à son tour les pièces de tissus elle échange rapidement avec REF sur les patrons (lignes 32-35), (Image 2). 


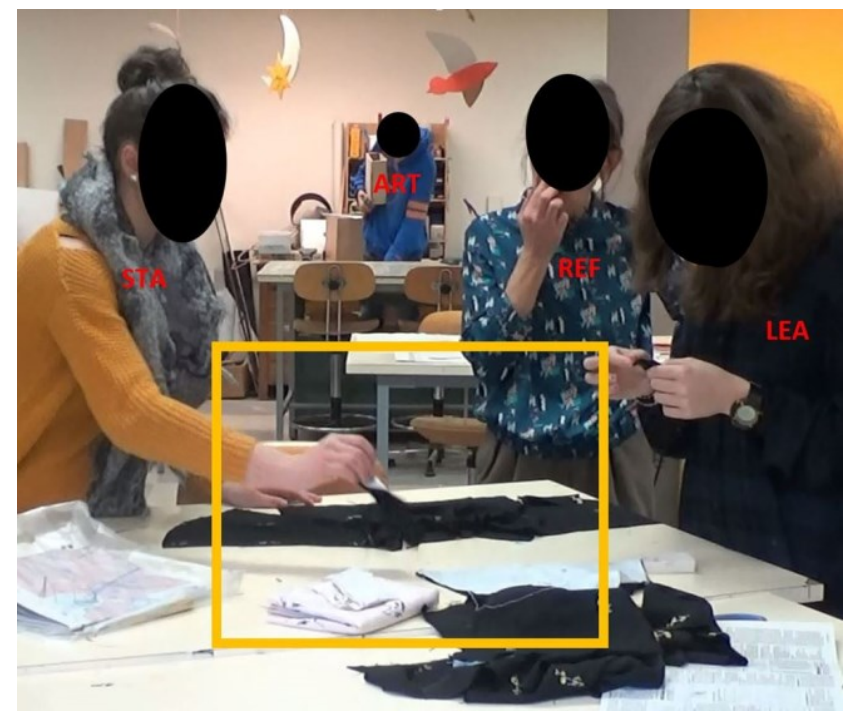

Image 2. Séquence d'observation B.

REF acquiesce (ligne 36) et va alors se tenir légèrement en retrait pendant 7 secondes, le temps que STA commence à manipuler les tissus pour préparer le travail de LEA et poursuivre l'ouvrage avec elle. Ceci se fait en présence de la REF, mais cette dernière n'intervient plus. Le fait qu'elle s'adresse à la ligne 38 à l'autre patient qui se trouve au fond de la salle marque son désengagement quant à la manipulation des tissus, à la fois auprès de la stagiaire et de la patiente LEA. C'est aussi un moment de transition vers la possibilité de réaliser une autre configuration, celle de la co-animation où la référente s'occupe de ART et la stagiaire de LEA.

\subsection{De l'observation à la co-animation à une opportunité de monstration et à la co-animation}

Nous parlons de co-animation lors d'une distribution des tâches distinctes mais complémentaires entre le tuteur et le stagiaire. Cette distribution peut être planifiée en amont ou être le résultat du déroulement situé et dynamique de l'activité. Dans nos données, les différentes configurations de participation au service de la formation comme l'observation, la co-animation ou encore la monstration et le laisser-faire ont des durées très variables pouvant aller de quelques secondes à plusieurs dizaines de minutes. Dans la majorité de cas, nous observons une transformation dynamique de ces configurations qui se réalisent dans l'action de manière contingente et peuvent rarement être anticipées. Nous proposons d'illustrer ce phénomène avec l'extrait suivant : «Couture_b » qui se déroule juste avant l'extrait précédent. STA et LEA travaillent ensemble et REF les observe tout en contrôlant l'installation d'un autre patient, ART, dans la salle. Au bout d'un moment elle s'éloigne de la table où se trouvent LEA et STA et s'adresse à ART. Ce faisant, elle quitte son rôle d'observatrice et initie une séquence de co-animation où la stagiaire peut travailler en autonomie (REF-ART/ STA-LEA). 


\section{Extrait 2 : «Couture_b»}

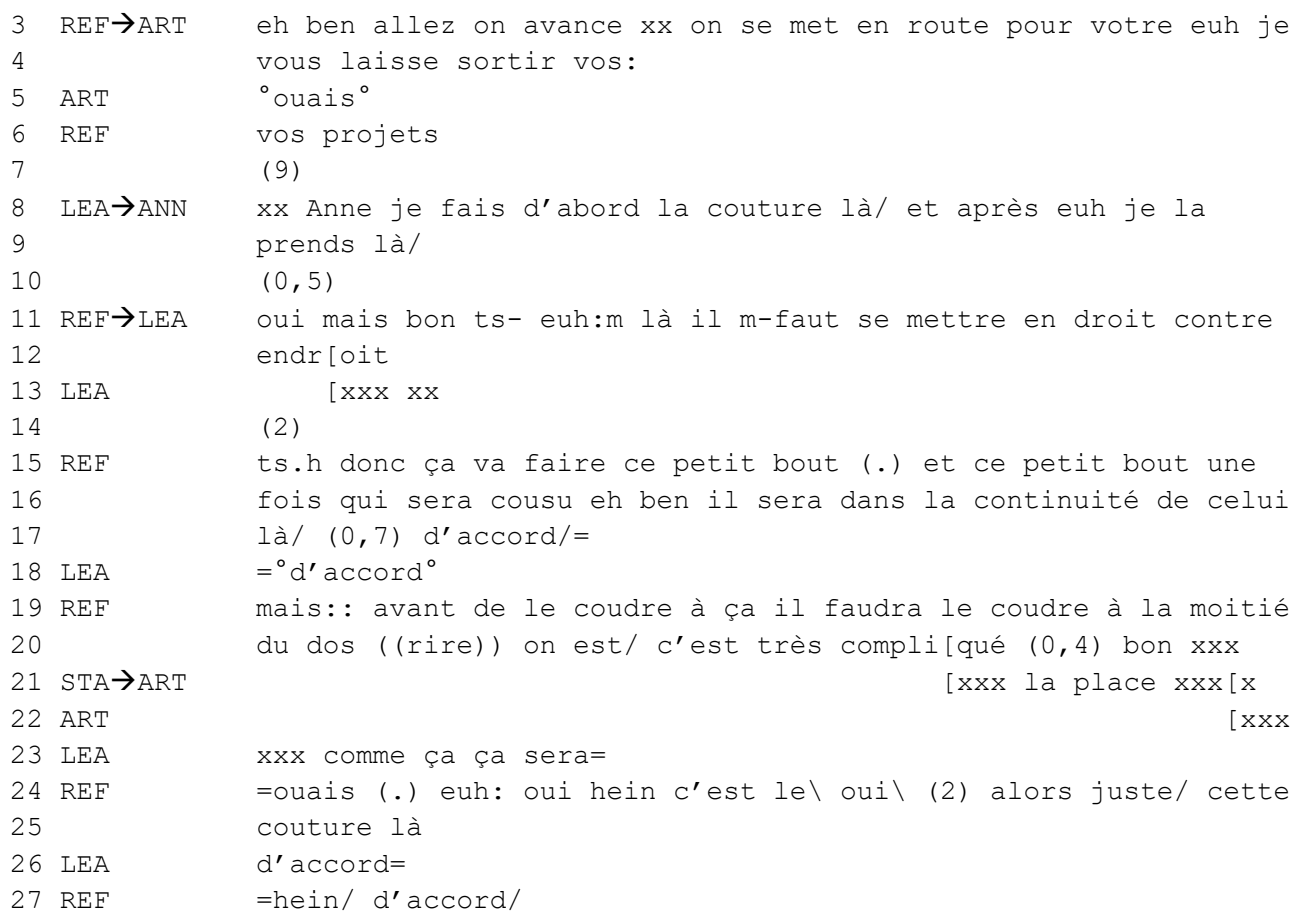

La séquence débute par l'installation d'ART dans la salle pour un atelier de menuiserie. Au début de l'extrait ART a pratiquement fini de mettre en place le matériel nécessaire. REF s'adresse alors à lui et lui propose de débuter son activité de menuiserie (lignes 3-6). À ce moment précis, elle arrête d'observer l'activité de STA avec LEA. En effet, pendant ce temps LEA manipule les tissus afin de confectionner une robe sous le regard attentif de STA. Une fois que ART est installé en salle de menuiserie et avant de démarrer une activité avec lui, REF se tourne vers STA et LEA, comme pour vérifier si tout va bien. LEA va alors interpeller la référente qui va se rapprocher de la table en lui posant une question technique (ligne 8). STA observe REF pendant quelques secondes en train de manipuler les tissus et donner des explications à LEA. Cette posture est typique dans les cas de monstration, où le stagiaire observe les gestes experts des professionnels expérimentés. De manière intéressante ce moment n'a pas été initié par la REF pour la STA mais par la patiente LEA. STA regarde ensuite à deux reprises vers la salle de menuiserie où se trouve ART et d'où on entend des bruits. Ce dernier semble avoir des difficultés avec le rangement du matériel de menuiserie. STA quitte la table où se trouvent LEA et REF et s'approche de ART pour lui proposer son aide (ligne 21). Une fois que REF a donné les explications à LEA, elle va regarder où se trouve STA. Après avoir observé que STA a pris en charge ART (Image 3), REF va se retourner vers LEA et continuer à échanger avec elle (ligne 24) (Image 4). 


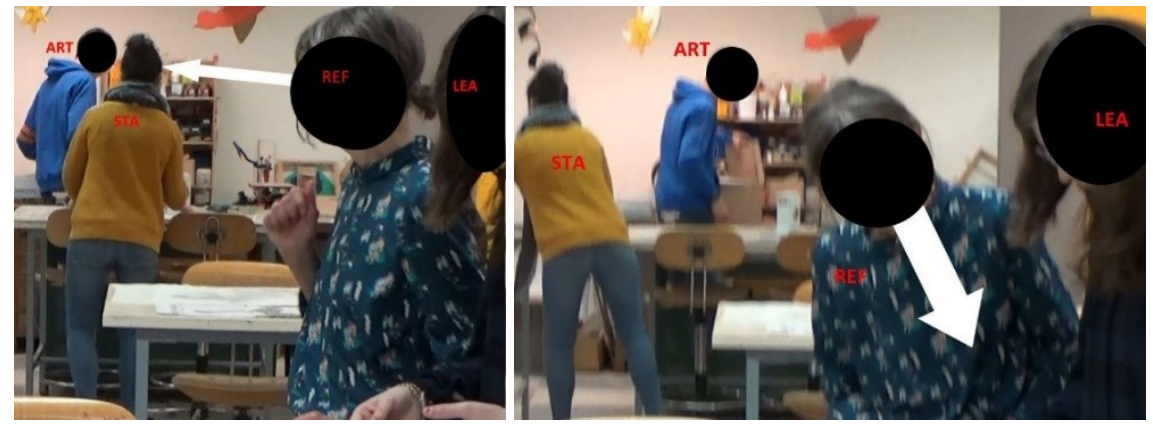

Images 3 et 4 . Configuration de co-animation.

Durant cette séquence, nous avons pu observer la transition d'une activité d'observation vers une activité de co-animation, transformée par l'intervention de LEA en une séquence de monstration puis en une séquence de co-animation afin de pouvoir répondre aux besoins des patients présents. Il n'est pas question ici d'activités formatives planifiées en amont mais plutôt d'opportunités de positionnements de la référente et de la stagiaire vis-à-vis des patients qu'elles saisissent à toutes fins pratiques.

Cet extrait illustre que dans le réel, même quand le tuteur observe, il n'est pas complétement en retrait et qu'aussi bien le stagiaire et les patients sont sensibles à sa présence et ses réactions. Des travaux sur la formation accompagnée des éducatrices de la petite enfance montrent que la posture d'observation est justement soumise aux imprévus du travail réel et que la professionnelle expérimentée qui endosse le rôle de tutrice est amenée à intervenir et co-animer une activité si la stagiaire l'interpelle (Markaki \& Rémery, 2013) ou si elle juge que cette dernière ne peut répondre seule aux exigences éducatives de l'activité menée (Filliettaz, 2015). Ici, nous constatons également l'importance des contingences du contexte et la forte orientation de la professionnelle et de la stagiaire vers les enjeux d'une prise en charge efficace des patients. Les configurations chargées d'enjeux formatifs se transforment de manière dynamique dans le temps, séquence d'actions par séquence d'actions. Les configurations interactionnelles observées dans nos données mettent aussi en évidence un monitoring continu de la part de la référente de l'ensemble de l'activité et des participants.

\subsection{La configuration de la monstration « corrective »}

La monstration résulte d'une activité du tuteur (dans l'exemple qui suit il s'agit d'une orthophoniste) qui souhaite rendre son activité professionnelle ostensible à la vue et la compréhension du stagiaire. Cette activité intervient alors que le stagiaire a entrepris une action que le référent souhaite corriger. C'est le stagiaire qui devient observateur et qui doit repérer les caractéristiques d'une activité professionnelle adéquate rendues visibles par cette intervention particulière de monstration. Ici, l'activité formatrice est la compréhension de la bonne pratique professionnelle. Cette configuration est illustrée par l'extrait $3:$ « Règles du jeu ». MAR et ALE sont des patients. MAR explique les règles d'un jeu de société qu'ils vont ensuite jouer tous ensemble. La stagiaire suit et ensuite participe à l'échange entre MAR et ALE. L'orthophoniste (ORT) et l'ergothérapeute (ER2) sont assis à côté et préparent le matériel du jeu.

\section{Extrait 3 : " règles du jeu "}

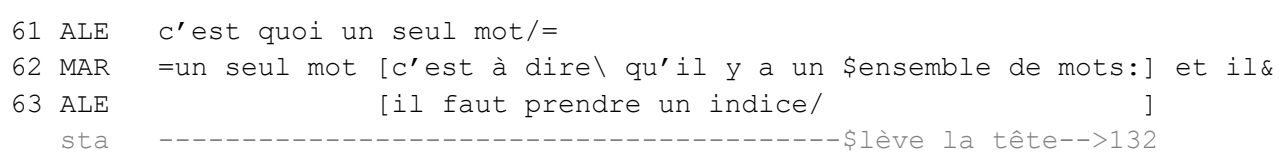




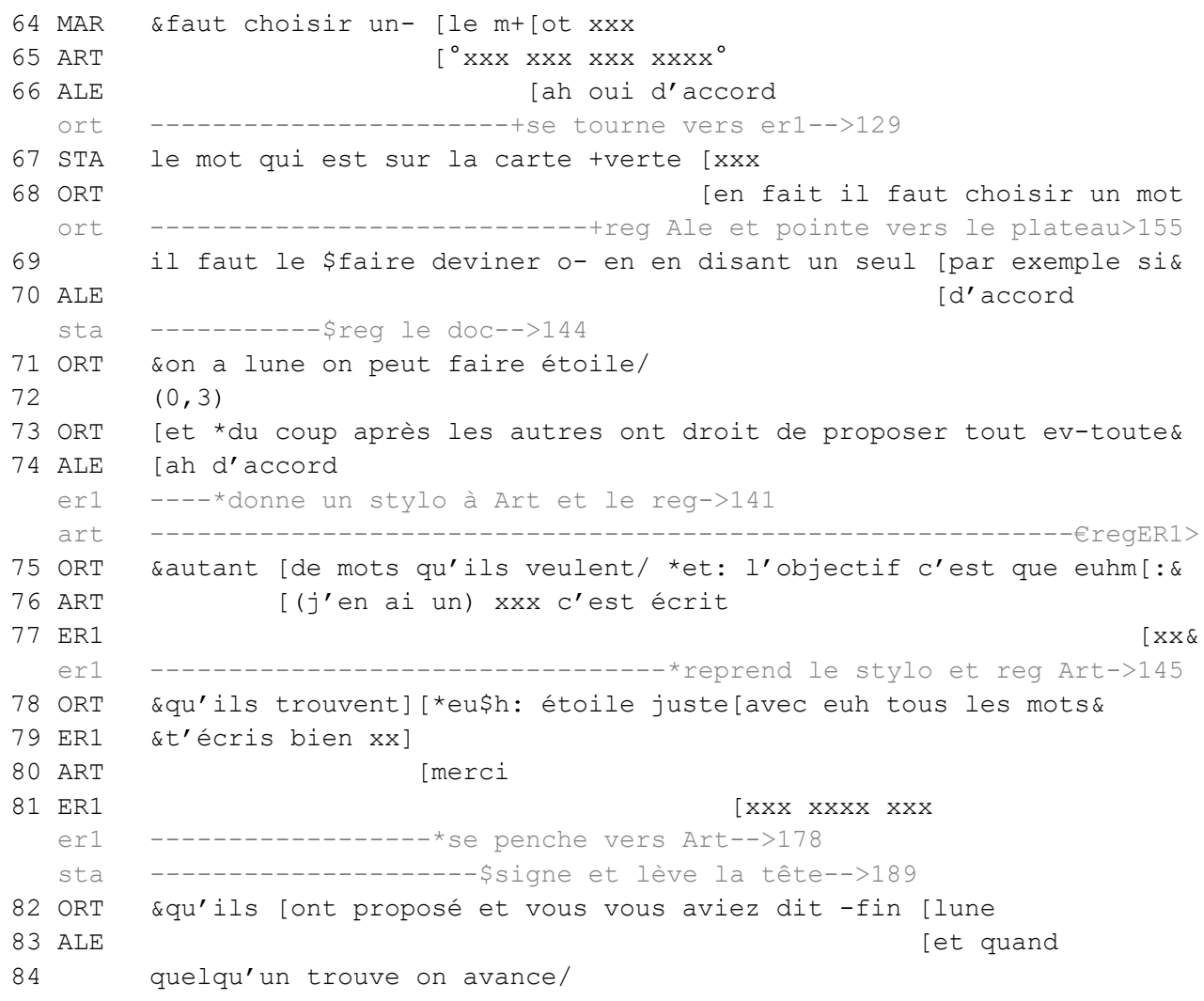

Dans cet extrait, STA intervient à la ligne 67 participant dès lors aux échanges entre MAR et ALE (lignes 61-66). Lors de son intervention, elle va regarder les professionnels (Image 5).

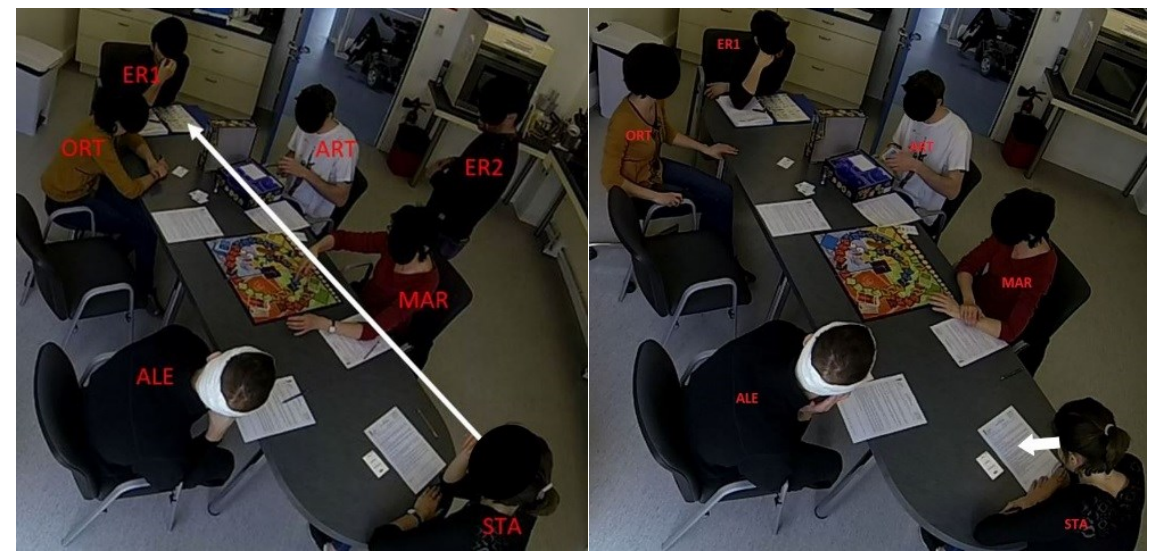

Images 5 et 6. Configuration de monstration

Cette intervention complète et tente probablement d'expliciter ce que dit MAR aux lignes 62 et 64 en réponse à la question d'ALE ( «c'est quoi un seul mot? ?, " il faut prendre un indice ?», 1.61 et 1.63). Avant que STA ait fini son énoncé, ORT intervient (image 6) à son tour, sans reprendre la proposition de la stagiaire, et complète ce que dit MAR en y associant des exemples et des gestes sur le plateau de jeu (à partir de la ligne 68). Ces exemples et gestes adressés à ALE pour comprendre le jeu, le sont aussi indirectement à STA. En ne 


\section{Extrait 5 : « menuiserie »}

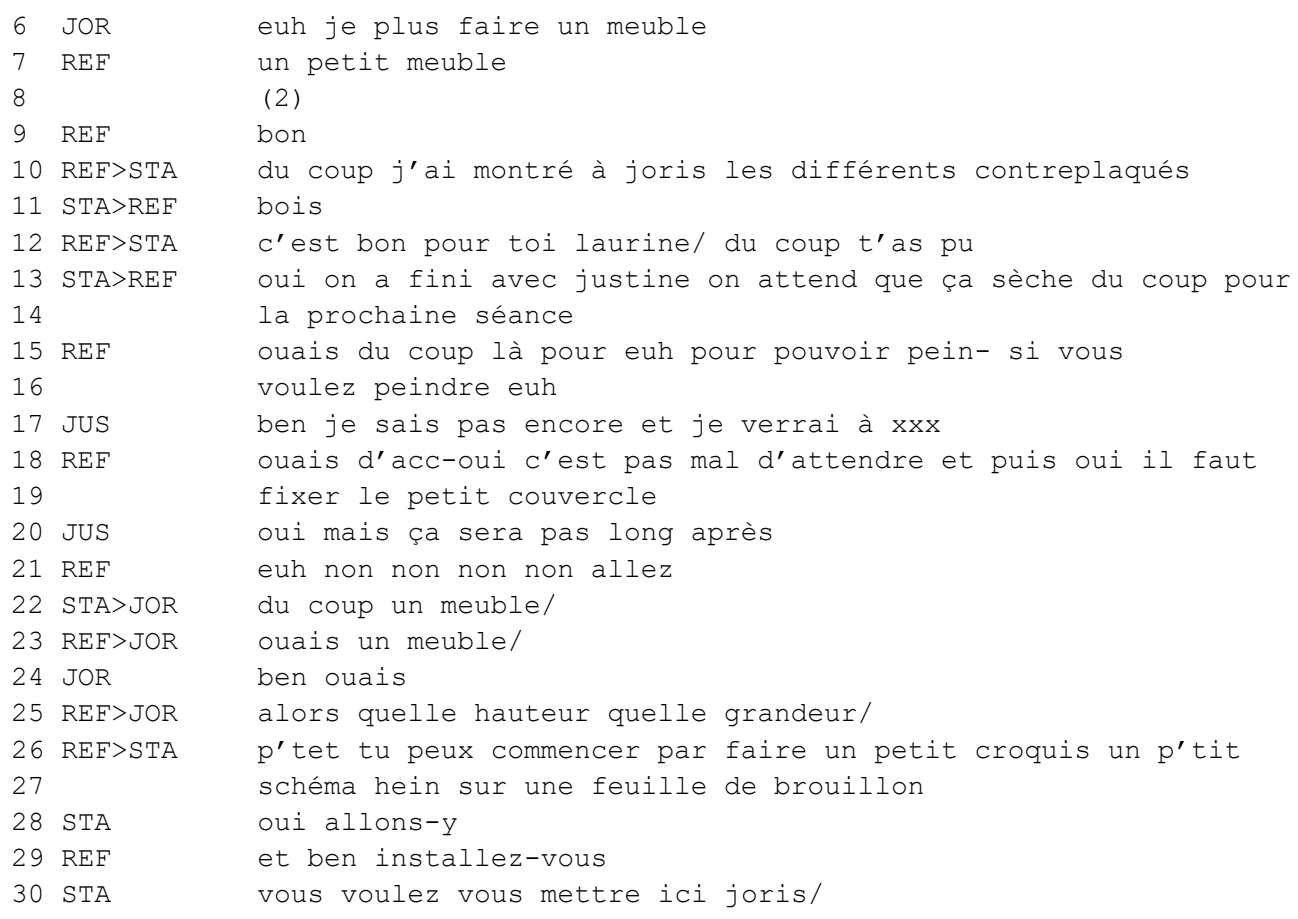

Dans cet extrait, la configuration de laisser faire est de nouveau initiée par REF (ligne 12 : «c'est bon pour toi/»). Par la suite, et avant que REF laisse STA et JOR seuls, la configuration ressemble beaucoup plus à une configuration de co-animation puisque STA et REF interagissent ensemble avec JOR et JUS (lignes 22-27).

Contrairement à l'extrait précédent, REF ne fait aucune allusion sur la durée pendant laquelle le patient sera avec la stagiaire et/ou si elle allait les rejoindre pour la suite de l'activité. REF va pendant ce temps sortir de la salle (Images 7 et 8 ).

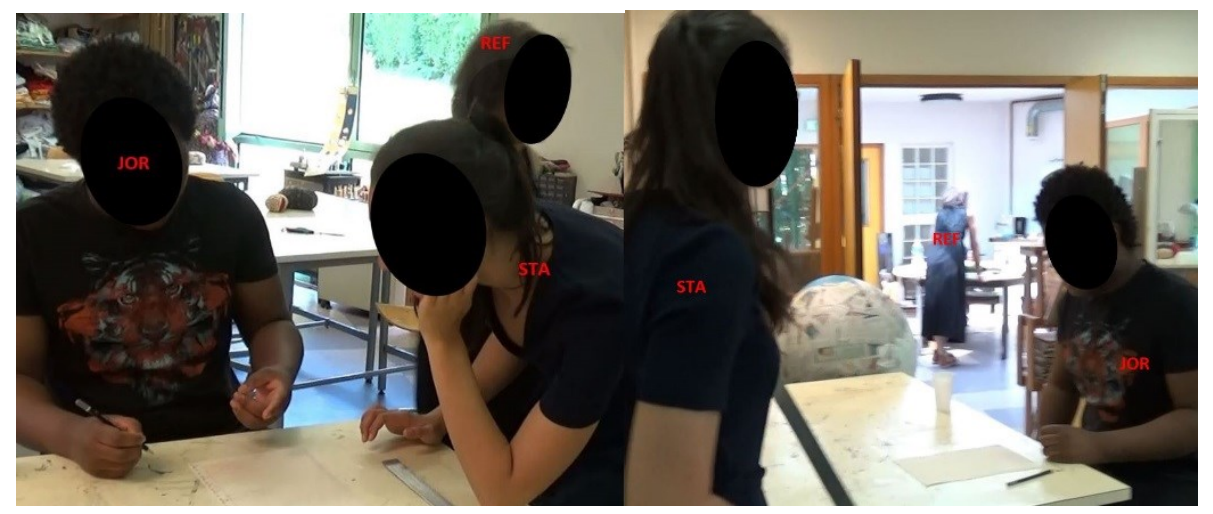

Images 7 et 8. Configuration de laisser faire.

Elle n'intervient que 6 minutes plus tard (Image 9) pour aider à placer une planche de bois. 


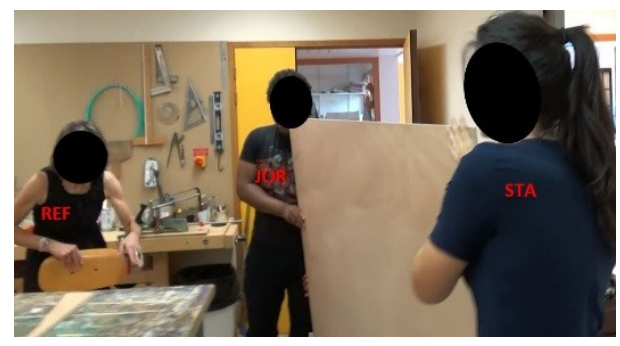

Image 9. Configuration de laisser faire.

De manière intéressante, même pendant cette configuration assumée pleinement par tous les participants, nous nous rendons compte que la référente reste suffisamment attentive à ce qui se passe pour proposer son aide quand cela est nécessaire lors du positionnement d'une planche de bois sur la table.

\section{Discussion}

Dans la formation d'ergothérapeutes et probablement aussi des autres professionnels des métiers de service à la personne, le tuteur est au centre d'une activité interactionnelle complexe avec plusieurs participants et aux finalités différentes. Nos données nous ont montré que les tuteurs s'orientent de manière différenciée vers ces finalités en fonction de l'activité, du stagiaire mais surtout en fonction des patients. Ces orientations se manifestent à travers les différentes configurations de participation entre les personnes présentes qui se transforment de manière dynamique et ajustée aux besoins des activités entreprises.

Dans nos entretiens, nous avions pu voir que les professionnels avaient des difficultés à différencier leurs actions auprès des stagiaires et des soins prodigués aux patients. Nos données nous montraient que dans le travail cette dissociation du rôle formateur et du rôle soignant était en effet vécue comme fort complexe.

Puis dans nos extraits, nous avions pu voir que la configuration de « laisser-faire » était systématiquement mise en place à la demande du tuteur. Pourrait-elle être mise en place à la demande du stagiaire ? Nous l'avons en effet remarqué parfois. Le stagiaire prend des initiatives d'interaction avec d'autres patients sans l'accord ou sans demander à son tuteur la possibilité de le faire (c'est le cas de la stagiaire dans l'extrait « couture_b »). Nous avons vu que ces initiatives de participation sont par la suite ratifiées ou corrigées par le tuteur de diverses manières comme dans l'extrait « règles du jeu ».

Il reste encore de nombreuses questions sans réponse, comme par exemple l'importance du choix du patient pour le stage quand cela est possible ou encore la préparation en amont des séances avec les patients. Ce travail vise à ouvrir un champ de recherche par l'analyse des interactions au travail des ergothérapeutes et interroge à la fois la nature des compétences interactionnelles des professionnels de santé et leur développement.

\section{Conclusion}

Les réformes pour l'universitarisation des métiers du champ sanitaire et médico-social en France ont eu pour conséquence de devoir se poser des questions importantes sur la qualité et les modalités de la formation. Ces questions devraient se poser sur la formation en école ou institut mais aussi sur l'importance des pratiques professionnelles des tuteurs de stage. La formation d'un professionnel de santé et en particulier l'ergothérapeute dans ce travail de recherche, ne consistait pas à la considérer comme une acquisition d'une liste de compétences 
d'un référentiel. C'est la mise en pratique de ces compétences dans un environnement changeant, d'adaptabilité au patient et aux situations de soin qui est primordiale. Le futur professionnel doit être formé à la complexité de l'interaction avec le patient. Comment alors le professionnel est-il formé à cette adaptabilité et à cette complexité d'interaction ?

Dans ce projet de recherche, nous avions souhaité nous concentrer sur l'analyse du travail réel pour explorer et documenter ces pratiques professionnelles. En ayant adopté une démarche inductive, nous avons pu questionner les pratiques interactionnelles complexes du tuteur de stage et la centration autour du ou des patients. Nos analyses du travail réel nous invitent à repenser le rôle formateur de l'ergothérapeute en situation de travail en mettant en évidence des configurations de participation chargées d'enjeux formatifs déterminées par la présence du patient. La prise en compte du patient dans les interactions est primordiale pour le tuteur, qui doit à la fois gérer des interactions soignantes en direction du patient et des interactions formatives en direction du stagiaire. La gestion de ces activités complexes requiert du tuteur des compétences spécifiques d'analyse et d'anticipation de ces interactions. Le tuteur doit ainsi savoir s'adapter à des activités variées avec le patient et/ou le stagiaire. Nous remarquons donc que la distinction entre les rôles (formateur/apprenant) et les activités (travailler/former) telles que décrites dans certains articles s'intéressant à la formation professionnelle est bien plus complexe et ne peut être réduite à une typologie avec des frontières étanches et établies à l'avance. La prise en compte du travail réel et donc des priorités du terrain, des urgences, des particularités des environnements professionnels doit être placée au centre des préoccupations lors de la conception des dispositifs de formation.

Cette complexité pointe également vers la question de la valorisation de ces activités professionnelles. La reconnaissance de la complexité de l'activité des ergothérapeutes et de l'expertise des tuteurs est essentielle pour les établissements qui participent à la formation de futurs professionnels. En accueillant régulièrement des stagiaires, ces établissements doivent pouvoir défendre leurs professionnels tuteurs en valorisant les compétences interactionnelles spécifiques à ces missions et en leur proposant un environnement de travail capacitant (Fernagu Oudet, 2012).

Et si au final la plus importante des compétences pour les professionnels de santé était la capacité à savoir interagir bien au-delà de l'expertise clinique ? Voici une question que nous souhaiterions voir traitée et discutée, non seulement en sciences humaines et sociales, mais également dans tous les milieux professionnels du soin et de l'accompagnement des patients, puisqu'elle concerne à la fois la valorisation de ces métiers au service de l'être humain mais aussi la formation de futurs professionnels.

\section{Références}

Filliettaz Laurent. (2015). Les ressources interactionnelles de la structuration des activités en contexte éducatif. Bulletin suisse de linguistique appliquée, vol. 101, p. 11-26.

Filliettaz Laurent, Durand Isabelle, Zogmal Marianne, Markaki Vassiliki. (2021). Le « schisme interactionnel », une ressource pour apprendre aux frontières du travail et de la formation professionnelle. Recherches en éducation, Université de Nantes.

Filliettaz Laurent, Rémery, Vanessa, Trébert Dominique. (2014). Relation tutorale et configurations de participation à l'interaction: Analyse de l'accompagnement des stagiaires dans le champ de la petite enfance. Activites, vol. $11 \mathrm{n}^{\circ} 1$. https://doi.org/10.4000/activites.288

Gosselin Claire, Dutil Elisabeth. (1995). Stimuler l'autonomie et la polyvalence des étudiants en ergothérapie: Description d'une expérience pédagogique et perception des professeurs. Canadian Journal of Occupational Therapy, vol. 62, n³, p. 127-137. 
Jovic Ljiljana, Goldszmidt Danielle, Monguillon Dominique. (2010). Encadrement des étudiants en stage, enseignement et recherche : Évaluation et valorisation des activités réalisées par des professionnels paramédicaux. Recherche en soins infirmiers, vol. 101, n², p. 81. https://doi.org/10.3917/rsi.101.0081

Kunegel Patrick. (2011). Les maîtres d'apprentissage : analyse des pratiques tutorales en situation de travail. Ed. L'harmattan (Coll. Action Et Savoir). ISBN : 2296561608

Markaki Vasiliki, Lelarge Anne-Eléonore. (à paraître). Négociation des territoires épistémiques et formation informelle en situation de travail. In : Rémery, V., Chrétien, F. \& Chatigny, C. (Ed.). Apprentissage et transmission de l'expérience en situation de travail : un dialogue entre ergonomie et formation d'adultes. Rouen : Presses Universitaires de Rouen et du Havre.

Markaki Vasiliki, Rémery Vanessa. (2013). Les processus de hiérarchisation dans l'activité tutorale au travail. Contribution au Congrès de l'Association Française de Sociologie, Nantes, 2-5 septembre 2013.

Fernagu Oudet Solveig. (2012). Chapitre 14. Favoriser un environnement « capacitant» dans les organisations. Dans : E. Bourgeois (éd.), Apprendre au travail, p. 201-213. Paris : Presses Universitaires de France.

Pekarek Doehler Simona. (2011) Approches interactionnistes de l'acquisition des langues étrangères : concepts, recherches, perspectives, Acquisition et interaction en langue étrangère [En ligne], 12 |2000, mis en ligne le 13 avril 2011, consulté le 01 mai 2021. URL : $\quad$ http://journals.openedition.org/aile/934; $\quad$ DOI : https://doi.org/10.4000/aile.934

Sacks Harvey, Schegloff Emmanuel, Jefferson Gail. (1974). A Simplest Systematics for the Organization of Turn Taking in Conversation. Language, vol. 50, p. 696-735. http://dx.doi.org/10.2307/412243

Sacks Harvey (1992). Lectures on Conversation. Edited by Gail Jefferson. Oxford : Basil Blackwell.

Schegloff Emmanuel, Sacks Harvey. (1973). 'Opening Up Closings', Semiotica, vol. 8, p. 289-327. https://doi.org/10.1515/semi.1973.8.4.289

Schegloff Emmanuel, Jefferson, Gail, Sacks, Harvey. (1977). The Preference for SelfCorrection in the Organization of Repair in Conversation. Language, vol. 53, n², p. 361. https://doi.org/10.2307/413107

Schegloff Emmanuel. (2007). Categories in action: Person-reference and membership categorization. Discourse Studies, vol. 9, $\mathrm{n}^{\circ} 4, \quad$ p. 433-461. https://doi.org/10.1177/1461445607079162

\section{Financements}

Ce travail a reçu le soutien financier de Initiatives de Recherches Stratégiques de l'Université Grenoble Alpes.

\section{Remerciements}

Nous remercions les ergothérapeutes, patients et stagiaires pour avoir accepté d'etre filmé, et les quatre ergothérapeutes pour les entretiens individuels. 